\title{
Formation Control of Underactuated Surface Vessels using the Null-Space-Based Behavioral Control
}

\author{
Filippo Arrichiello Stefano Chiaverini \\ Dipartimento di Automazione, Elettromagnetismo, \\ Ingegneria dell'Informazione e Matematica Industriale \\ Università degli Studi di Cassino \\ via G. Di Biasio 43, 03043 Cassino (FR), Italy \\ ff.arrichiello, chiaverini\}eunicas.it
}

\author{
Thor I. Fossen \\ Department of Engineering Cybernetics \\ Norwegian University of Science and Technology \\ NO-7491 Trondheim, Norway \\ fossen@ieee.org
}

\begin{abstract}
In this paper the application of a behavior-based control approach, namely the Null-Space-based Behavioral control, to coordinate a fleet of autonomous surface vessels is presented. The NSB can be considered as a centralized guidance system aimed at driving the fleet in complex environments while simultaneously performing multiple tasks, i.e., obstacle avoidance or keeping a formation. In order to apply the guidance system to a fleet of underactuated surface vessels, the NSB works in combination with a low-level maneuvering control that, taking care of the dynamics of the vessels, elaborates the motion commands to generate the generalized forces at the actuators. The guidance system has been simulated in the accomplishment of a mission in presence of obstacles and sea current in the environment.
\end{abstract}

\section{INTRODUCTION}

In the latest years the field of cooperation and coordination of multi-robot systems has been subject of considerable research efforts. The main motivations are that multi-robot systems can perform tasks more efficiently than a single robot or can accomplish tasks not executable by a single one. Moreover, multi-robot systems have advantages like increasing tolerance to possible vehicle fault, providing flexibility to the task execution or taking advantage of distributed sensing and actuation. Multi-robot systems can be composed by different kind of autonomous vehicles like Unmanned Grounded Vehicles (UGV), Unmanned Aerial Vehicles (UAV), Unmanned Underwater Vehicles (UUV) or autonomous surface vessels. In this paper, the case of multi-robot systems made up of autonomous surface vessels is considered.

When controlling a fleet of autonomous surface vessel, a common requirement is the motion coordination among the vessels; that is, the vessels have to move in the environment keeping a suitable relative configuration. Thus, coordination and formation control of autonomous surface vessel have been subject of research in the last few years [1], [2], [3]. Among other possible methods, in this paper a behavior-based approach to coordinate the fleet is proposed.

Behavior-based approaches, widely studied for mobile robotics applications [4], are useful to guide a multi-robot system in an unknown or dynamically changing environment. These approaches give the system the autonomy to navigate in complex environments avoiding low-level path planning, using sensors to obtain instantaneous information of the environment and increasing flexibility of the system. Among the behavioral approaches, seminal works are reported in the papers [5] and [6], while the textbook [4] offers a comprehensive state of the art. The behavior-based approach proposed in this paper, namely the Null-Space-based Behavioral approach (NSB), differs from the other existing methods in the behavioral coordination method, i.e., in the way the outputs of the single elementary behaviors are assembled to compose a complex behavior [7].

From a marine applications point of view, the NSB can be seen as a centralized guidance system aimed at coordinating the motion of the fleet in different scenarios and to achieve different missions. Using a suitable policy to manage multiple tasks, the NSB elaborates the instantaneous motion references for each vessel. How to follow these motion references, instead, is the aim of a low-level maneuvering control system that, on the basis of kinematical and dynamical characteristics of the vessels, has to elaborate the generalized forces applied by the actuators. Thus, the maneuvering control has to steer a vessel along a desired path and make it moving with a desired velocity [8]. For fully actuated ships, the maneuvering control problem is extensively explained in [8], [9], while for underactuated ships, equipped with less than 3 actuators (i.e., only 1-2 actuators are used to control the surge, sway and yaw modes), the maneuvering control still is a challenging problem.

The decomposition of the controller in two stages (NSB and maneuvering) makes possible to decouple the problems of coordination and maneuvering and to deal with them separately. The guidance system has been simulated while performing with a fleet of underactuated vessels different navigation missions in presence of obstacles and sea current in the environment; the results of one simulation are reported to show the effectiveness of the proposed approach.

\section{GUIDANCE SYSTEM FOR UNDERACTUATED SURFACE VESSELS}

The guidance system proposed in this paper has to solve simultaneously different problems: it is responsible for ships' safety, that is, it has to make the ships able to avoid static and dynamic obstacles doing a dynamic path generation (path generation on-the-fly); it has to achieve different kinds of 
missions, e.g., navigation keeping a fixed relative formation or changing the route or the formation considering weather and environmental conditions; it has to maneuver underactuated ships (ships equipped with less than 3 actuators).

To this purpose, the guidance system is decomposed in two main blocks: the Null-Space-based Behavioral control and the maneuvering control (see Figure 1). The NSB takes into consideration the parameter of the mission, the environmental condition and the status of the fleet to elaborate the desired velocities for each vehicle. These velocities represent the reference input for the maneuvering controls that, taking into consideration kinematics and dynamics of the ship, have to define the generalized forces applied by the actuators. Extension and more details of the proposed technique can be found in [10].

\section{A. Null-Space-based Behavioral control for autonomous ve- hicles}

The Null-Space-based Behavioral approach is a centralized system aimed at guiding a platoon of generic autonomous vehicles in different scenarios and to achieve different missions. Following the main behavioral approaches, the mission of the platoon is decomposed in elementary tasks, e.g., move the barycenter of the platoon, avoid obstacles, keep a formation. For each task, a function that measures its degree of fulfilment (e.g., a cost or a potential function) can be defined; thus, in a static environment, the task is achieved when its output is constant at a value that minimizes the task function. Following the techniques inherited from inverse kinematics for industrial manipulators described in [11], [12], the NSB uses a hierarchybased logic to combine multiple conflicting tasks. In particular, the NSB is able to fulfill or partially fulfill each task according to their position in the hierarchy and according to the eventual conflicts with the highest-priority tasks.

With reference to a generic platoon of autonomous vehicles, the basic concepts of the NSB are recalled in the following.

By defining as $\sigma \in \mathbb{R}^{m}$ the task variable to be controlled and as $\boldsymbol{p} \in \mathbb{R}^{n}$ the system configuration, it is:

$$
\boldsymbol{\sigma}=\boldsymbol{f}(\boldsymbol{p})
$$

with the corresponding differential relationship:

$$
\dot{\boldsymbol{\sigma}}=\frac{\partial \boldsymbol{f}(\boldsymbol{p})}{\partial \boldsymbol{p}} \boldsymbol{v}=\boldsymbol{J}(\boldsymbol{p}) \boldsymbol{v}
$$

where $\boldsymbol{J} \in \mathbb{R}^{m \times n}$ is the configuration-dependent task Jacobian matrix and $\boldsymbol{v} \in \mathbb{R}^{n}$ is the system velocity. Notice that $n$ depends on the specific autonomous system considered and the term system configuration simply refers to the vessel position/orientation (in case of a material point $n=2$, in case of a single surface vessel $n=3$, in the case of a platoon of $z$ surface vessels $n=3 z$ ).

An effective way to generate motion references $\boldsymbol{p}_{d}(t)$ for the vehicles, starting from desired values $\sigma_{d}(t)$ of the task function, is to act at the differential level by inverting the (locally linear) mapping (2); in fact, this problem has been widely studied in robotics (see, e.g., [13] for a tutorial).
A typical requirement is to pursue minimum-norm velocity, leading to the least-squares solution

$$
\boldsymbol{v}_{d}=\boldsymbol{J}^{\dagger} \dot{\boldsymbol{\sigma}}_{d}
$$

where the (pseudo)inverse $\boldsymbol{J}^{\dagger}$ must be properly computed according to the dimension and the rank of the task Jacobian matrix.

At this point, the vehicle motion controller needs a reference position trajectory besides the velocity reference; this can be obtained by time integration of $\boldsymbol{v}_{d}$. However, discrete-time integration of the vehicle's reference velocity would result in a numerical drift of the reconstructed vehicle's position; the drift can be counteracted by a so-called Closed Loop Inverse Kinematics (CLIK) version of the algorithm, namely,

$$
\boldsymbol{v}_{d}=\boldsymbol{J}^{\dagger}\left(\dot{\boldsymbol{\sigma}}_{d}+\boldsymbol{\Lambda} \tilde{\boldsymbol{\sigma}}\right)
$$

where $\boldsymbol{\Lambda}$ is a suitable constant positive-definite matrix of gains and $\widetilde{\sigma}$ is the task error defined as $\widetilde{\sigma}=\sigma_{d}-\boldsymbol{\sigma}$.

It is worth noting that the Null-Space-based Behavioral control intrinsically requires a differentiable analytic expression of the tasks defined so that it is possible to compute the required Jacobians.

Considering the case of multiple tasks, on the analogy of eq. (4) the single task velocity is computed as

$$
\boldsymbol{v}_{i}=\boldsymbol{J}_{i}^{\dagger}\left(\dot{\boldsymbol{\sigma}}_{i, d}+\boldsymbol{\Lambda}_{i} \tilde{\boldsymbol{\sigma}}_{i}\right)
$$

where the subscript $i$ denotes $i$-th task quantities. If the subscript $i$ also denotes the degree of priority of the task (i.e., Task 1 is the highest-priority one), in the a case of 3 tasks, according to [14] and denoting with $\boldsymbol{I}$ the identity matrix of proper dimension, the CLIK solution (4) is modified into

$$
\boldsymbol{v}_{d}=\boldsymbol{v}_{1}+\left(\boldsymbol{I}-\boldsymbol{J}_{1}^{\dagger} \boldsymbol{J}_{1}\right)\left[\boldsymbol{v}_{2}+\left(\boldsymbol{I}-\boldsymbol{J}_{2}^{\dagger} \boldsymbol{J}_{2}\right) \boldsymbol{v}_{3}\right] \text {. }
$$

Remarkably, eq. (6) has a nice geometrical interpretation. Each task velocity is computed as if it were acting alone; then, before adding its contribution to the overall vehicle velocity, the $i$-th-priority task is projected (by $\boldsymbol{I}-\boldsymbol{J}_{i-1}^{\dagger} \boldsymbol{J}_{i-1}$ ) onto the null space of the immediately higher-priority task so as to remove those velocity components that would conflict with it.

The Null-Space-based Behavioral control always fulfils the highest-priority task at nonsingular configurations. The lowerpriority tasks, on the other hand, are fulfilled only in a subspace where they do not conflict with the ones having higher priority, that is, each task reaches a sub-optimal condition that optimizes the task respecting the constraints imposed by the highest-priority tasks.

\section{B. Maneuvering control of underactuated ships}

In this paper the case of underactuated ships, equipped with a single screw propeller and a rudder is considered. The actuators are acting only in the surge and yaw direction, thus the following 3-DOF nonlinear maneuvering model [8], is considered:

$$
\begin{aligned}
\dot{\boldsymbol{\eta}} & =\boldsymbol{R}(\psi) \boldsymbol{\nu} \\
\boldsymbol{M} \dot{\boldsymbol{\nu}}+\boldsymbol{N} \boldsymbol{\nu} & =\left[\begin{array}{c}
\tau_{1} \\
0 \\
\tau_{3}
\end{array}\right]+\boldsymbol{R}^{\mathrm{T}}(\psi) \boldsymbol{w}
\end{aligned}
$$




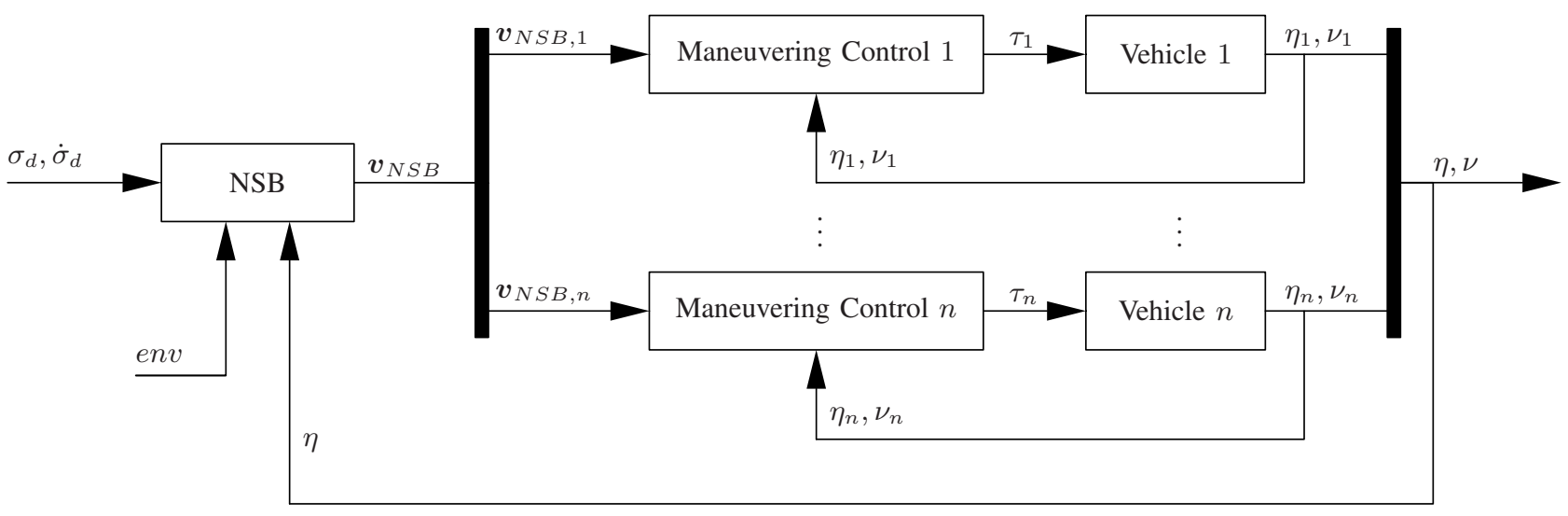

Fig. 1. Sketch of the guidance system.

where $\boldsymbol{\eta}=[n e \psi]^{\mathrm{T}}$ is the position and attitude vector in the North-East-Down (NED) reference frame; $\boldsymbol{\nu}=[u v r]^{\mathrm{T}}$ is the linear and angular velocity vector in the body-fixed reference system; $\boldsymbol{R}(\psi)$ is a rotation matrix; $\boldsymbol{M}$ is the matrix of inertial parameters defined as

$$
\boldsymbol{M}=\left[\begin{array}{ccc}
m_{11} & 0 & 0 \\
0 & m_{22} & m_{23} \\
0 & m_{32} & m_{33}
\end{array}\right] ;
$$

$N$ is the damping matrix

$$
\mathbf{N}=\left[\begin{array}{ccc}
n_{11} & 0 & 0 \\
0 & n_{22} & n_{23} \\
0 & n_{32} & n_{33}
\end{array}\right]
$$

$\tau_{1}$ and $\tau_{3}$ are respectively the force in surge direction and torque in the yaw direction; $\boldsymbol{w}$ is the vector of the environmental forces (wind, currents, etc.) acting on the ship in the NED reference system.

In the proposed guidance system, the maneuvering control is aimed at making each vehicle to follow its velocity reference command elaborated by the NSB. According to the NSB characteristics, the reference command are dynamically changing and, to regulate the velocity, the following control low is applied:

$$
\begin{aligned}
U_{d} & =U_{N S B} \cdot \frac{1+\cos \left(\chi_{N S B}-\chi\right)}{2} \\
\tau_{1} & =k_{p 1}\left(U_{d}-U\right)+k_{i 1} \int_{0}^{t}\left(U_{d}-U\right) d t \\
\tau_{3} & =k_{p 3}\left(\chi_{N S B}-\chi\right)+k_{i 3} \int_{0}^{t}\left(\chi_{N S B}-\chi\right) d t-k_{d 3} \dot{\chi}
\end{aligned}
$$

where, following the reference system in Figure 2, the velocity of the ship is represented by the norm of the vector $U$ and its course angle $\chi$ respect to the North direction; $U_{N S B}$ and $\chi_{N S B}$ are desired values elaborated by the NSB, and the $k_{*}$ 's are proper PID gains.

\section{TASKS}

According to the behavioral control approach, the mission of the fleet is decomposed in three elementary tasks: move

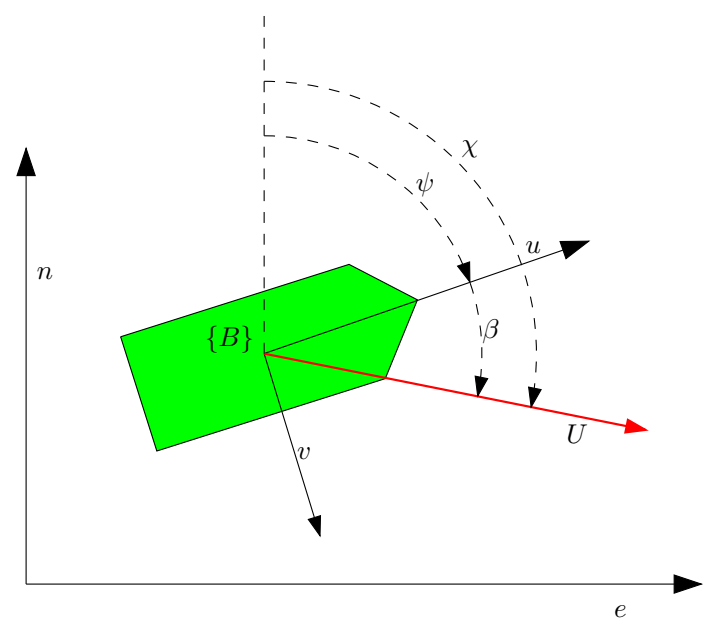

Fig. 2. Reference frames and relevant variables for the surface vessel. $\chi$ is the course angle, $\psi$ is the heading angle and $\beta$ is the sideslip angle

the barycenter of the fleet, keep a formation relative to the barycenter, and avoid collisions with obstacles and among vehicles. In this section, the corresponding task functions are presented.

\section{A. Barycenter}

The barycenter of a platoon expresses the mean value of the vehicles positions. In a 2-dimensional case (like for surface vessels) the task function is expressed by:

$$
\boldsymbol{\sigma}_{b}=\boldsymbol{f}_{b}\left(p_{1}, \ldots, p_{n}\right)=\frac{1}{n} \sum_{i=1}^{n} \boldsymbol{p}_{i}
$$

where $\boldsymbol{p}_{i}=\left[\eta_{i, 1} \eta_{i, 2}\right]^{\mathrm{T}}$ is the position of the vehicle $i$.

Deriving the previous relation with respect to time gives

$$
\dot{\boldsymbol{\sigma}}_{b}=\sum_{i=1}^{n} \frac{\partial \boldsymbol{f}_{b}(\boldsymbol{p})}{\partial \boldsymbol{p}_{i}} \boldsymbol{v}_{i}=\boldsymbol{J}_{b}(\boldsymbol{p}) \boldsymbol{v},
$$

where the Jacobian matrix $\boldsymbol{J}_{b} \in \mathbb{R}^{2 \times 2 n}$ is

$$
\boldsymbol{J}_{b}=\frac{1}{n}\left[\begin{array}{llll} 
& 1 & 0 & \\
\cdots & 0 & 1 & \cdots
\end{array}\right] \text {. }
$$


Following eq. 4 , the output of the barycenter task function is

$$
\boldsymbol{v}_{b}=\boldsymbol{J}_{b}^{\dagger}\left(\dot{\boldsymbol{\sigma}}_{b, d}+\boldsymbol{\Lambda}_{b} \widetilde{\boldsymbol{\sigma}}_{b}\right)
$$

where the desired value of the task function represent the desired trajectory of the barycenter.

\section{B. Rigid Formation}

The rigid formation task moves the vehicles to a predefined formation relative to the barycenter. The task function is defined as:

$$
\boldsymbol{\sigma}_{f}=\left[\begin{array}{c}
\boldsymbol{p}_{1}-\boldsymbol{p}_{b} \\
\vdots \\
\boldsymbol{p}_{n}-\boldsymbol{p}_{b}
\end{array}\right],
$$

where $\boldsymbol{p}_{i}$ are the coordinates of the vehicle $i$ and $\boldsymbol{p}_{b}=\boldsymbol{\sigma}_{b}$ are the coordinates of the barycenter. Writing for simplicity the vector $\boldsymbol{p}$ as $\left[\eta_{1,1} \ldots \eta_{n, 1} \eta_{1,2} \ldots \eta_{n, 2}\right]^{\mathrm{T}}$ then the Jacobian matrix $\boldsymbol{J}_{f} \in \mathbb{R}^{2 n \times 2 n}$ is:

$$
\boldsymbol{J}_{f}=\left[\begin{array}{ll}
\boldsymbol{A} & \boldsymbol{O} \\
\boldsymbol{O} & \boldsymbol{A}
\end{array}\right],
$$

where $\boldsymbol{A} \in \mathbb{R}^{n \times n}$ is:

$$
\boldsymbol{A}=\left[\begin{array}{cccc}
1-\frac{1}{n} & -\frac{1}{n} & \ldots & -\frac{1}{n} \\
-\frac{1}{n} & 1-\frac{1}{n} & \ldots & -\frac{1}{n} \\
\vdots & \vdots & \ddots & \vdots \\
-\frac{1}{n} & -\frac{1}{n} & \ldots & 1-\frac{1}{n}
\end{array}\right]
$$

The desired value $\sigma_{f, d}$ of the task function describes the shape of the desired formation; that is, once defined the formation, the elements of $\sigma_{f, d}$ represent the coordinates of each vehicle in the barycenter reference frame.

The output of the formation task function, in the case of fixed desired formation $\left(\dot{\boldsymbol{\sigma}}_{f, d}=\mathbf{0}\right)$, is:

$$
\boldsymbol{v}_{f}=\boldsymbol{J}_{f}^{\dagger} \boldsymbol{\Lambda}_{f} \widetilde{\boldsymbol{\sigma}}_{f}
$$

\section{Obstacle avoidance}

The obstacle avoidance task function is built individually to each vehicle, i.e., it is not an aggregate task function. In fact, an obstacle in the environment may be close to some vehicle but far from some other; moreover, each vehicle is an obstacle for the others in the team but not for itself.

With reference to the generic vehicle in the team, in presence of an obstacle in the advancing direction, the task function has to elaborate a driving velocity, aligned to the vehicle-obstacle direction, that keeps the vehicle at a safe distance $d$ from the obstacle. Therefore, it is:

$$
\begin{aligned}
\sigma_{o} & =\left\|\boldsymbol{p}-\boldsymbol{p}_{o}\right\| \\
\sigma_{o, d} & =d \\
\boldsymbol{J}_{o} & =\hat{\boldsymbol{r}}^{\mathrm{T}},
\end{aligned}
$$

where $\boldsymbol{p}_{o}$ is the obstacle position and

$$
\hat{\boldsymbol{r}}=\frac{\boldsymbol{p}-\boldsymbol{p}_{o}}{\left\|\boldsymbol{p}-\boldsymbol{p}_{o}\right\|}
$$

is the unit vector aligned with the obstacle-to-vehicle direction. According to the above choice, eq. (5) simplifies to

$$
\boldsymbol{v}_{o}=\boldsymbol{J}_{o}^{\dagger} \lambda_{o} \widetilde{\boldsymbol{\sigma}}_{o}=\lambda_{o}\left(d-\left\|\boldsymbol{p}-\boldsymbol{p}_{o}\right\|\right) \hat{\boldsymbol{r}} \text {. }
$$

It is worth noting that, being

$$
\mathcal{N}\left(\boldsymbol{J}_{o}\right)=\boldsymbol{I}-\hat{\boldsymbol{r}} \hat{\boldsymbol{r}}^{\mathrm{T}},
$$

the tasks of lower priority than the obstacle avoidance are only allowed to produce motion components tangent to the circle of radius $d$ and centered in $\boldsymbol{p}_{o}$, so as to not interfere with the enforcement of the safe distance $d$. The comparison with the main behavioral approaches of the proposed technique while achieving a move-to-goal mission with obstacle avoidance with a single vehicle can be found in [7].

While the implementation of the proposed obstacleavoidance task function is the same for both punctual environmental obstacles and other vehicles, in the case of continuous obstacles it changes a bit. In particular, for convex- or straightline obstacles, $\boldsymbol{p}_{o}$ represents the coordinates of the closest point of the obstacle to the ship at the current time instant.

In the frequent case of multiple obstacles acting simultaneously (e.g., both an obstacle in the environment and the other vehicles of the team) a priority among their avoidance should be defined; a reasonable choice is to assign the currently closest obstacle the highest priority. In critical situations the obstacle avoidance function may give a null-velocity output; this causes delay to the mission or loss of vehicles to the formation but increases safety of the approach.

\section{Simulation CASE STUdy}

In this Section simulation results of a fleet of vessels navigating under the proposed control scheme in presence of obstacles in the environment are presented. In the considered scenario a fleet of 8 underactuated vessels has to move its barycenter along a rectilinear path, keeping a circular formation and avoiding 2 line-shaped obstacles, while an environmental force (due to sea current and wind) is acting on the ships.

To accomplish the mission the following three elementary tasks are identified: obstacle avoidance (avoid collisions with other vessels and environmental obstacles), barycenter (move the barycenter of the fleet along a desired path) and rigid formation (specifically, keep a circular formation). The obstacle avoidance is always the highest-priority task because its achievement is of crucial importance to preserve integrity of the vehicles, while the barycenter and the rigid formation are the secondary and tertiary tasks, respectively.

The obstacle avoidance task function has to ensure each vessel a safe distance of $15 \mathrm{~m}$ from the environmental obstacles and from other vessels. For each vessel, the task function is activated only when the distance from environmental obstacles or other vessels becomes lower than $15 \mathrm{~m}$. When the vessel is simultaneously close to (i.e., under $15 \mathrm{~m}$ from) multiple obstacles, then the closest obstacle has the highest priority. Since a moving obstacle is assumed to be more dangerous than a fixed one, if the vessel is simultaneously close to an environmental obstacle and another vessel, the avoidance of the other vessel takes higher priority if the distance from it 

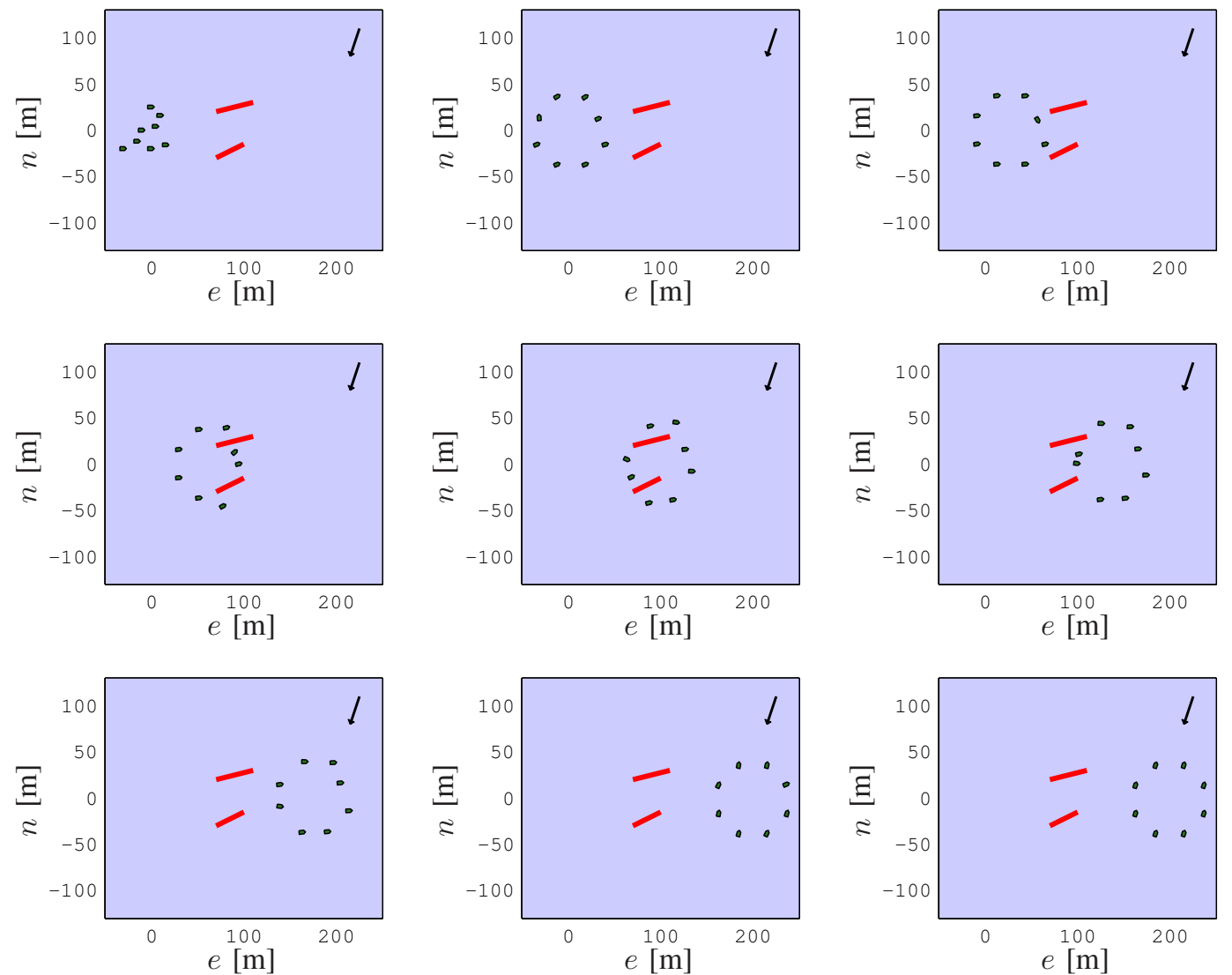

Fig. 3. Nine snapshots of the mission execution. A fleet of 8 underactuated vessels has to move its barycenter along a rectilinear path while keeping a circular formation and avoiding 2 line-shaped obstacles in presence of an environmental force (whose direction is represented by the arrow).

is greater than the distance from the environmental obstacle multiplied by 0.3 .

The desired trajectory of the barycenter is a rectilinear segment that connects the initial position $\left[\begin{array}{lll}0 & 0\end{array}\right]^{\mathrm{T}} \mathrm{m}$ to the final position $[2000]^{\mathrm{T}} \mathrm{m}$ according to a fifth-order polynomial time law of $80 \mathrm{~s}$ duration with null initial and final velocity. The gain matrix of the barycenter task function is a 2-dimensional identity matrix.

The vessels have to attain and keep a circular formation of radius $40 \mathrm{~m}$ around the barycenter. The positions of the vessels in the circular formation are defined by an optimization algorithm that takes care of the instantaneous configuration. The gain matrix of the rigid formation task function is a 16dimensional identity matrix.

The vessels dynamics obeys to the model described in Subsection II-B, where the matrices $M$ and $N$ in SI units are:

$\boldsymbol{M}=\left[\begin{array}{ccc}25.8 & 0 & 0 \\ 0 & 33.8 & 1.01 \\ 0 & 1.01 & 2.76\end{array}\right] \quad \boldsymbol{N}=\left[\begin{array}{ccc}2 & 0 & 0 \\ 0 & 7 & 0.1 \\ 0 & 0.1 & 0.5\end{array}\right]$

The parameters of the maneuvering control are $k_{p 1}=40$, $k_{i 1}=0, k_{p 2}=10, k_{i 2}=0.1$ and $k_{d 2}=0.5$. The environmental force due to sea current and wind is supposed constant to the value $\boldsymbol{w}=[-10-30]^{\mathrm{T}} \mathrm{N}$.
Figures 3 and 4 show that, starting from an initial random configuration, the ships attain the desired circular configuration before reaching the obstacles; close to the obstacles, the desired configuration is released to avoid hitting and, after passing over, they attain again the desired formation. Notice that to keep the final configuration, the ships orient themselves in the opposite direction to the environmental force; this placement confirms the effectiveness of the interaction between the NSB and the maneuvering control.

Figures 5, 6 and 7 show the tracking errors of the task functions during the whole mission. The obstacle avoidance is activated only in the critical phases of the mission where changes of formation occur, i.e., close to the obstacles and while the ships, starting from a random configuration, attain the circular formation. The barycenter task function tracking error starts from a low value (i.e., the barycenter of the initial configuration is quite close to its desired location), increases during avoidance of the line-shaped obstacles and decreases once they are overtaken; while the choice of a null $\boldsymbol{K}_{i 1}$ brings to a non-null final error, it avoids oscillations of the ships' position around the final configuration. The tracking error of the circular formation task function starts from a large value due to the initial misplacement of the ships; it then quickly decreases when the ships initially attain the desired configuration, increases during the obstacle avoidance phase 


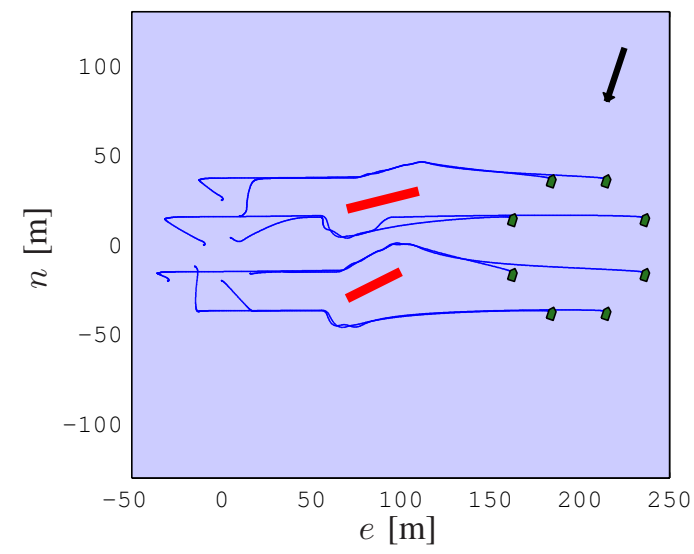

Fig. 4. Paths followed by the ships during the mission.

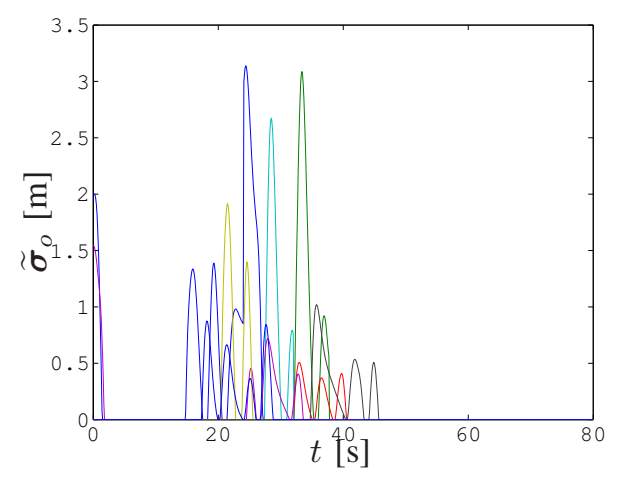

Fig. 5. Tracking error of the obstacle avoidance task function.

and converges to zero once passed the obstacles. Video of the performed simulation can be found at: http : //webuser.unicas.it/arrichiello/video/

\section{CONCLUSiON}

In this paper the Null-Space-based Behavioral control has been presented to guide a fleet of autonomous underactuated surface vessels in complex environments. The NSB works in combination with the low-level maneuvering controls of each ship to take into consideration the dynamics of the fleet. The guidance system has been simulated in a complex mission involving the attainment of a formation while moving through obstacles in presence of sea current; the obtained results show the effectiveness of the proposed method.

\section{REFERENCES}

[1] R. Skjetne, S. Moi, and T. Fossen, "Nonlinear formation control of marine craft," in Proceedings 41st IEEE Conference on Decision and Control, Las Vegas, Nevada, USA, 2002, pp. 1699-1704.

[2] I.-A. F. Ihle, R. Skjetne, and T. Fossen, "Nonlinear formation control of marine craft with experimental results," in Proceedings 43rd IEEE Conference on Decision and Control, vol. 1, Paradise Island, The Bahamas, Dec 2004, pp. 680-685.

[3] I.-A. F. Ihle, J. Jouffroy, and T. Fossen, "Formation control of marine surface craft using lagrange multipliers," in Proceedings 44th IEEE Conference on Decision and Control and European Control Conference, Seville, Spain, 2005.

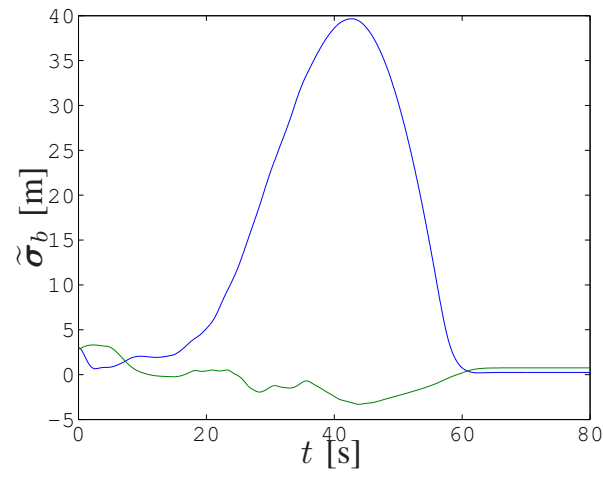

Fig. 6. Tracking error of the barycenter task function.

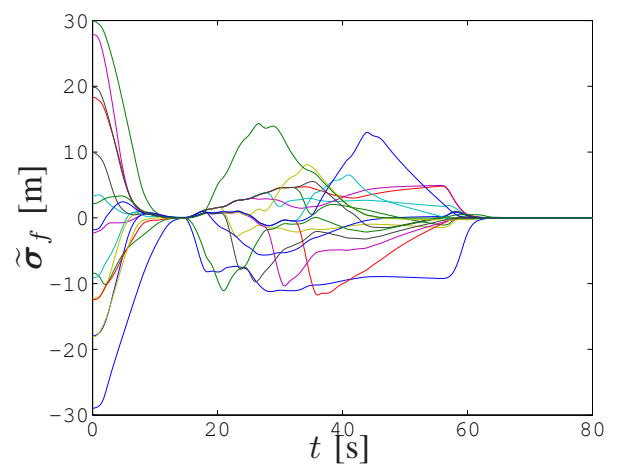

Fig. 7. Tracking error of the circular formation task function.

[4] R. Arkin, Behavior-Based Robotics. Cambridge, MA: The MIT Press, 1998.

[5] R. Brooks, "A robust layered control system for a mobile robot," IEEE Journal of Robotics and Automation, vol. 2, pp. 14-23, 1986.

[6] R. Arkin, "Motor schema based mobile robot navigation," The International Journal of Robotics Research, vol. 8, no. 4, pp. 92-112, 1989.

[7] G. Antonelli, F. Arrichiello, and S. Chiaverini, "Experimental kinematic comparison of behavioral approaches for mobile robots," in Proceedings 16th IFAC World Congress, Prague, CZ, July 2005.

[8] T. Fossen, Marine Control Systems: Guidance, Navigation and Control of Ships, Rigs and Underwater Vehicles. Trondheim, Norway: Marine Cybernetics, 2002.

[9] R. Skjetne, The Maneuvering Problem. Trondheim, Norway: PhD.Thesis, Norwegian University of Science and Technology, 2005.

[10] F. Arrichiello, S. Chiaverini, and T. Fossen, Formation control of Marine Surface Vessels using the Null-Space-based Behavioral Control. Group Coordination and Cooperative Control, Springer-Verlag's Lecture Notes in Control and Information Sciences series, May 2006, pp. 1-19.

[11] B. Bishop, "On the use of redundant manipulator techniques for control of platoons of cooperating robotic vehicles," IEEE Transactions on Systems, Man and Cybernetics, vol. 33, no. 5, pp. 608-615, Sept. 2003.

[12] G. Antonelli and S. Chiaverini, "Kinematic control of a platoon of autonomous vehicles," in Proceedings 2003 IEEE International Conference on Robotics and Automation, Taipei, TW, Sept. 2003, pp. 1464-1469.

[13] B. Siciliano, "Kinematic control of redundant robot manipulators: A tutorial," Journal of Intelligent Robotic Systems, vol. 3, pp. 201-212, 1990.

[14] S. Chiaverini, "Singularity-robust task-priority redundancy resolution for real-time kinematic control of robot manipulators," IEEE Transactions on Robotics and Automation, vol. 13, no. 3, pp. 398-410, 1997. 